\title{
Filigrane
}

Écoutes psychothérapiques

\section{L'horizon oedipien des blessures narcissiques identitaires}

\section{Christian Seulin}

Volume 19, numéro 1, printemps 2010

Adieu Oedipe, bonjour Narcisse?

URI : https://id.erudit.org/iderudit/044178ar

DOI : https://doi.org/10.7202/044178ar

Aller au sommaire du numéro

Éditeur(s)

Revue Santé mentale au Québec

ISSN

1192-1412 (imprimé)

1911-4656 (numérique)

Découvrir la revue

Citer cet article

Seulin, C. (2010). L'horizon oedipien des blessures narcissiques identitaires. Filigrane, 19(1), 39-48. https://doi.org/10.7202/044178ar

\section{Résumé de l'article}

L'auteur rappelle la conception freudienne du complexe d'Oedipe comme structure universelle pour la mettre en question en regard de la clinique contemporaine et avancer l'idée du rôle central de l'organisateur oedipien de l'analyste dans la cure. Les fantasmes s'articulent au complexe d'Oedipe et constituent un monde à propos duquel Freud a émis des opinions contradictoires qui laissent entrevoir la nécessité d'une acquisition individuelle de la structure oedipienne. Une situation clinique de cure psychanalytique chez une patiente ayant vécu des situations traumatiques d'abandon montre que chez certains patients aux blessures narcissiques identitaires sévères, l'organisation oedipienne déjà présente et sans doute activée dans la cure permet une réinterprétation des traumatismes précoces et se révèle une structure attractive pour la psyché. 


\title{
L’horizon œdipien des blessures narcissiques identitaires
}

\author{
Christian Seulin
}

L'auteur rappelle la conception freudienne du complexe d'ÆEdipe comme structure universelle pour la mettre en question en regard de la clinique contemporaine et avancer l'idée du rôle central de l'organisateur œedipien de l'analyste dans la cure. Les fantasmes s'articulent au complexe d'CFdipe et constituent un monde à propos duquel Freud a émis des opinions contradictoires qui laissent entrevoir la nécessité d'une acquisition individuelle de la structure œdipienne. Une situation clinique de cure psychanalytique chez une patiente ayant vécu des situations traumatiques d'abandon montre que chez certains patients aux blessures narcissiques identitaires sévères, l'organisation œdipienne déjà présente et sans doute activée dans la cure permet une réinterprétation des traumatismes précoces et se révèle une structure attractive pour la psyché.

\begin{abstract}
ujourd'hui, notre pratique quotidienne d'analyste nous confronte fréquemment à des patients souffrant de problématiques narcissiques identitaires dans lesquelles le facteur traumatique tient une place de premier rang. Ces configurations cliniques interrogent la place du complexe d'Edipe dans la clinique contemporaine mais aussi dans la culture. Cette évolution de la clinique met effectivement en question le point de vue de Freud concernant le complexe d'Edipe qu'il considérait comme une structure immuable et universelle. Dans un certain nombre de cas, les blessures narcissiques qui tenaient le devant de la scène analytique ne représentent qu'une entrave sérieuse au déploiement d'un complexe d'CEdipe pourtant présent. L'enjeu de la cure sera alors de restaurer des conditions topiques et économiques permettant l'assomption de la dynamique œdipienne.
\end{abstract}

\section{Pérennité et universalité du complexe d'夭́dipe?}

Une lecture des écrits de Freud sur le complexe d'CEdipe (Seulin, 2007) met en lumière la fonction structurelle fondamentale dans la psyché humaine de ce complexe. Pour Freud, le complexe d'Edipe est universel, transmis phylogénétiquement, tel un schème indépendant des organisations psychopathologiques. On peut, par exemple se référer à ce qu'il écrit dans sa célèbre observation de l'homme aux loups: «Nous pouvons souvent remarquer que le schème remporte la victoire sur l'expérience de vie individuelle, ainsi lorsque, dans notre cas, le père devient castrateur et celui qui menace la sexualité enfantine, malgré un complexe d'Edipe par ailleurs inversé» (À partir de l'histoire d'une névrose infantile, 1918, p.116). Il s'agit donc 
pour Freud d'une structure présente chez chaque être humain et qui prime sur le vécu singulier de l'individu. Freud écrit en 1920 «Chaque nouvel arrivant dans le monde humain est mis en devoir de venir à bout du complexe d'Edipe » puis ajoute un peu plus loin qu'il considère la reconnaissance du complexe d'Edipe comme «le schibboleth qui distingue les partisans de la psychanalyse de leurs adversaires » (note de 1920 ajoutée aux Trois essais sur la sexualité p.170).

Que peut-on penser aujourd'hui de l'hypothèse freudienne d'une transmission dans le ça tant du complexe d'CEdipe que des fantasmes originaires et de la préhistoire de l'humanité? La fiction anthropologique freudienne de Totem et tabou a-t-elle encore une certaine pertinence? Ne faut-il pas rapporter ces idées freudiennes aux effets de la culture comme aux influences des quelques générations précédentes, ce qui relativise la portée d'une hypothétique transmission phylogénétique et amène à prendre en compte la relative fragilité des structures psychiques transmises, la menace qui pèse sur elles? La question demeure cependant au sujet de la position freudienne. Comment comprendre que Freud ait fait du complexe d'Edipe un invariant? Tout être humain est certes confronté à l'Edipe mais nous devons bien constater que ce complexe n'a pas toujours chez les patients la fonction organisatrice que Freud lui conférait. Il est possible que cet invariant concernant le complexe d'Edipe ait été en lien avec la préoccupation chez Freud d'une objectivation scientifique des découvertes de la psychanalyse. Il faut aussi rappeler son souci, tout au long de son œuvre de différencier la psychanalyse de la suggestion et d'asseoir une connaissance dégagée de toute influence. Mais il me paraît surtout nécessaire de prendre en compte le rôle du complexe d'Edipe comme organisateur de la théorie et de la pratique analytique. Sa place dans le contre-transfert de l'analyste est également fondamentale et servira de vecteur pour l'évolution œdipienne des analysants.

La clinique comme les théories analytiques ont évolué. Nos analysants semblent plus souvent aux prises avec une problématique narcissique qui occulte la configuration œdipienne. À moins qu'il ne s'agisse aussi de l'évolution du regard de l'analyste sur sa clinique, ce, en rapport avec les changements sociétaux, reflets d'une évolution ou d'une involution culturelle dans laquelle l'organisation odipienne s'avère menacée.

Un certain nombre d'auteurs ont, avec justesse, mis l'accent sur la place du tiers dans la naissance du moi et des fantasmes, mais aussi comme premier stade ou prémice de l'organisation œdipienne. Ainsi, Michel Fain (1971) a proposé la métaphore de la censure de l'amante, quand la mère se détourne du bébé pour redevenir femme désirante du père de l'enfant avec les effets psychiques en rapport chez l'infans. Claude Le Guen avec le concept d'Édipe originaire (1974) a décrit l'inscription de la triangulation et la place du «non mère» comme première figure du père. D. W. Winnicott, d'une certaine façon, lorsqu'il évoque la destruction de l'objet corollaire de la naissance du fantasme (1971) et le passage de l'objet subjectif à l'objet objectif désigne la place du tiers. André Green de son côté, avec la théorie généralisée du tiers substituable (1984) montre la place incontournable de l'objet de l'objet. Nous sommes là aux sources de ce qui donnera dans sa forme achevée, élaborée et 
fantasmée, l'organisation œdipienne. Cependant, à mon sens, ces travaux montrent une orientation des recherches vers les sources ontogénétiques du complexe d'CEdipe et étayent l'idée d'une conquête individuelle avec ses aléas, même si la triangulation est une contrainte psychique de l'humain. La place incontournable du tiers ne suffit cependant pas à l'organisation œdipienne car il existe des triangulations sans CEdipe dans lesquelles les places ne sont pas définies ou bien les critères de différenciation échappent aux repères fondamentaux que sont la différence des sexes et des générations.

L'évolution sociétale et technologique permet à court terme d'envisager des modalités de procréation dans lesquelles, grâce à l'utérus artificiel, la présence d'une mère génitrice ne sera plus nécessaire. Quel risque pèsera dès lors sur les possibilités de développement d'une organisation triangulée et à plus forte raison œedipienne? On peut penser que c'est très tôt, dès les premiers échanges voire au cours du développement fotal que s'implantent les premières bases de ce qui ouvrira à la vie fantasmatique et à l'Edipe. Les sujets issus de tels modes de procréation surmonteront-ils le traumatisme qui consiste à sortir d'une machine pour trouver une mère avec sa vie psychique nécessairement étrangère?

\section{Brève remarque sur les fantasmes}

Le complexe d'Edipe et les fantasmes sont au cour de la sexualité infantile, ellemême au centre de la cure analytique. Les diverses déclinaisons fantasmatiques reposent sur les fantasmes originaires qui tous se constituent comme des réponses théoriques aux énigmes posées à l'enfant ainsi que l'ont montré Laplanche et Pontalis (1964). Ces fantasmes originaires s'articulent au complexe d'CEdipe.

Dans l'œuvre de Freud, deux paradoxes semblent repérables au sujet des fantasmes qui mettent en lumière les périls pesant sur eux et par voie de conséquence sur l'organisation œdipienne. Le premier est issu de la confrontation de deux points de vue divergents. En 1911 (Formulations sur les deux principes du cours des événements psychiques), Freud considère que le monde des fantasmes est clivé, indépendant de l'épreuve de réalité, uniquement soumis au principe de plaisir et qu'il ne s'étaye pas sur des objets réels. En 1924 (La perte de la réalité dans la névrose et dans la psychose), Freud utilise le mot de réserve pour parler du monde fantasmatique mais il écrit que ce monde maintient un contact avec le moi et contient des traces d'un passé réel satisfaisant. Entre la fermeture à la réalité extérieure et l'ouverture à cette réalité, se dessine une opposition nous montrant, me semble-t-il, que le monde fantasmatique est menacé de deux côtés. D’une part du côté de la fermeture à la réalité par le délire, d'autre part du côté de l'ouverture sur la réalité, donc sur la perception, par la pure répétition de traces perceptives. Ces considérations mettent l'accent sur l'importance dynamique des échanges entre dedans et dehors, leur enrichissement mutuel et montrent que le fantasme est une conquête de la vie psychique.

Le second paradoxe freudien est le suivant. Pour Freud, la naissance du fantasme nécessite, au contraire de la conception kleinienne, un certain degré d'organisation topique. Il faut en effet la précondition de l'expérience de satisfaction et de sa 
répétition hallucinatoire. Mais, en même temps, à l'opposé, Freud ne cesse de soutenir le caractère structurel, phylogénétiquement transmis des fantasmes originaires et de l'Edipe.

C'est peut-être dans ce double paradoxe à propos des fantasmes que se situerait la vulnérabilité de la structure œdipienne. Structure préexistante, il lui faut néanmoins pour advenir une organisation topique, elle-même dépendante des conditions environnementales, celles des parents puis de la société et de la culture.

\section{Blessures narcissiques identitaires et $\widetilde{E}$ dipe}

Lors de la rencontre analytique, un nombre important de patients nous montre que le complexe d'Edipe ne semble pas efficient dans leur fonctionnement psychique. Je soulignerai cependant la pertinence de ne pas opposer l'organisation œdipienne aux pathologies narcissiques et de penser les situations cliniques comme faisant coexister Edipe et Narcisse selon des degrés et des modes d'organisation variables. Il convient de souligner l'hétérogénéité de fonctionnement psychique des analysants et par là même prendre en compte le poids des mécanismes de déni et de clivage coexistant avec le refoulement et responsables de registres distincts de fonctionnement. Dans ces conjonctures cliniques, le travail analytique va d'abord souvent viser à restaurer une organisation topique, un inconscient dynamique et un préconscient à même de faire émerger du sens. La restauration en question ne saurait concerner les retrouvailles avec un fonctionnement antérieur du patient, dont l'analyste ne sait rien, mais vise bien plutôt à favoriser chez l'analysant un fonctionnement mental au plus près du modèle de la première topique freudienne. Cette restauration touche à l'inconscient dynamique et au préconscient de façon indissociable car les processus primaire et secondaire constituent un couple fonctionnel dont chaque terme suppose la fonctionnalité de l'autre. La réorganisation du refoulement ira alors de pair avec la capacité de rêver comme de fantasmer chez un analysant auparavant en difficulté pour la régression formelle, dont la cure a souvent débuté dans la douleur d'une régression à la dépendance et dans le drame de l'actualisation traumatique. À l'opposé de la cure classique et d'ailleurs très théorique du névrosé dans laquelle la configuration œdipienne organisatrice est un pré-requis, le rôle organisateur du complexe d'œdipe sera dans ces cas le fruit bénéfique d'une cure engagée sous les auspices d'une problématique narcissique et traumatique. L'enjeu de ces cures est donc de restaurer ou d'instaurer une organisation oedipienne suffisamment dégagée du poids des traumatismes narcissiques et identitaires. Ce travail va nécessairement solliciter les organisateurs psychiques de l'analyste qui se manifestent dans son cadre et sa pratique mais aussi dans son contre-transfert et ses théories. À ce titre, le complexe d'CEdipe et le surmoi post-œdipien de l'analyste vont pouvoir jouer un rôle dans la restauration d'un fonctionnement œedipien chez l'analysant. Cependant, toutes les configurations cliniques dominées par l'atteinte narcissique ne sont pas identiques. Nous pourrions de façon schématique différencier deux types de figures cliniques. Les situations dans lesquelles manifestement le complexe d'Edipe n'a pas été suffisamment organisateur dans l'environnement précoce 
du patient. Dans ces cas, ce que certains ont pu nommer « œdipification» est une conquête difficile de la cure qui n'est pas toujours ni souhaitable ni accessible, car le risque d'induction d'un faux-self existe. L'autre cas de figure, de bien meilleur augure, est constitué par des situations où les facteurs traumatiques ont en quelque sorte occulté et tenu à l'écart, pour des raisons économiques, une préorganisation œdipienne bien présente et inscrite dans l'environnement premier. Dans la cure de ces cas, l'Edipe fonctionne comme un attracteur (Michel Ody, 1989) qui permet une nouvelle mise en sens des traumatismes précoces. Dans ces situations, qui au fond doivent être apparentées après-coup aux névroses, il faut prendre en considération le rôle contre-transférentiel de l'organisateur psychique œedipien de l'analyste qui viendra renforcer l'attraction de cette structure chez le patient. C'est l'une de ces situations cliniques que je proposerai comme illustration.

\section{De l'abandon à I'CEdipe}

Sophie, âgée de 40 ans, est une femme soignée et élégante au visage triste et au maintien rigide. Elle est mariée et mère de deux enfants. Sa demande d'analyse reposait sur un mal être diffus où pouvaient se repérer d'intenses angoisses de séparation. Cultivée, exerçant non sans difficultés un métier à fortes responsabilités, elle me semblait mettre en œuvre de puissantes défenses par le déni, l'effacement évacuateur et réprimer fortement ses affects qu'elle neutralisait ainsi. Ces défenses qui s'exprimaient entre autre par des attaques du cadre commencent à se relâcher alors que nous sommes dans la quatrième année de son analyse.

Très vite au début de la cure, Sophie a apporté un rêve d'angoisse dans lequel une maison s'effondrait, détruite par un tremblement de terre ainsi qu'un souvenir écran. Dans son souvenir, elle a 2 ou 3 ans, est au lit et sa mère est près d'elle. Elle tient la main de sa mère. Elle s'endort puis s'éveille brusquement, sa mère a disparu. Elle la cherche en vain, en proie à un puissant sentiment de détresse.

De son enfance, elle a peu de souvenirs. Sophie se rappelle de violentes disputes entre ses parents quand elle était petite. Elle était terrifiée. Ses souvenirs n'acquièrent une certaine continuité qu'après ses 10 ans, moment de la naissance d'un frère.

Enfant, elle n'a cessé de changer de lieu de résidence et de pays du fait des fonctions professionnelles de son père. À de nombreuses reprises, elle a été séparée de ses parents, parfois pour des périodes de plusieurs mois. Elle vivait alors en France le plus souvent chez une tante maternelle très aimée.

Dans cette quatrième année d'analyse, Sophie tente de retrouver les souvenirs de son enfance en Australie entre 4 et 10 ans. Au fil des séances, quelques souvenirs émergent de sa gouvernante, de ses jouets, de fêtes d'école ou d'anniversaire. Mais elle n'a pas de souvenir des parents ni de sa vie au quotidien. Un sentiment de malaise aussi puissant qu'indéfinissable se relie à une longue séparation d'avec ses parents. Ces derniers étaient partis en vacances deux mois faire le tour de l'Australie tandis que Sophie avait été envoyée seule en France chez ses grands parents. Avec difficulté, elle parviendra à situer ce séjour à l'âge de 9 ans et en hiver, ce qui correspondait aux grandes vacances dans l'hémisphère sud. Sa mère a accouché 9 mois plus tard. Sophie 
pense que cette période a correspondu à la conception de son frère. Qu'a-t-elle éprouvé lors de cette séparation? Elle a dû en souffrir, que lui a-t-on dit ou expliqué? Lui revient le sentiment pénible d'ennui, de solitude et de froideur chez ses grands-parents.

\section{Deux séances}

À la séance, Sophie apporte un rêve : elle est dans une vieille maison. Il y a plusieurs étages. Une grande réception doit avoir lieu mais elle n'y est pas invitée. Elle part et cherche à acheter un vêtement, une veste de peau dans une boutique qu'elle connaît. La veste ne lui va pas, elle est moche sur elle, elle ne l'achète pas.

Sophie associe sur une série d'événements récents. La réception à laquelle elle n'est pas conviée lui fait penser au repas qu'elle a organisé la semaine précédente pour ses parents. C'était en même temps la fête de sa mère et Sophie lui a offert un joli cadeau. C'était une bonne soirée et ses parents ont invité Sophie, son mari et leurs enfants à venir les retrouver pour le week-end suivant à la campagne. Sophie n'a pas répondu immédiatement, aussi sa mère lui a proposé de leur téléphoner. Sophie ne l'a fait qu'avant de partir le samedi matin et n'a pu alors joindre ni son père ni sa mère. Elle a vécu cela comme un "lâchage», comme une invitation de pure forme, «factice». Au fond, me dit-elle, «mes parents s'en fichaient que nous venions». Malgré l'impossibilité de joindre les parents, elle s'est rendue le samedi à la maison de campagne. Ils n'y étaient pas mais en se promenant dans les environs, Sophie est tombée sur eux. Au détour d'un chemin, elle a vu ses deux parents vêtus de tenues de jogging noires. C'était comme si elle «ne les avait jamais vus, comme des étrangers ». Dans la séance, Sophie est envahie d'affects de colère, de tristesse et de déception. Elle me dit qu'elle s'était fait une joie à l'idée d'une partie de tennis avec son père et que, comme d'habitude, il ne s'est pas intéressé à elle, elle s'est sentie "transparente » pour lui. Je note intérieurement pour moi que Sophie attendait surtout de ce week-end un lien privilégié avec son père.

Les circonstances particulières de ce rendez-vous manqué avec les parents font songer que Sophie, par une forme discrète de répétition agie, s'est mise en position de revivre activement un abandon traumatique subi autrefois passivement par des parents vécus comme des étrangers. En effet, Sophie n'a tenté de contacter ses parents qu'au dernier moment, avant de partir, et, comme elle ne s'était pas annoncée avant, il est probable que les parents ne comptaient plus sur sa venue.

Sophie poursuit les associations sur son rêve et la veste de peau la conduit à penser à un manteau qu'elle s'est acheté il y a peu de temps avec l'argent que les parents lui ont donné pour son anniversaire. Elle se plaint: "Ils ne me font pas de cadeau mais me donnent de l'argent, à charge pour moi de m'acheter ce qui me plaît!» L'amertume la domine.

À la séance du lendemain, Sophie revient d'abord longuement sur sa colère par rapport aux parents, surtout sa mère, puis elle me raconte que sa fille de 13 ans lui a parlé de la mort de Paul. Elle lui a dit combien elle avait été affectée par ce décès, qu'elle aimait beaucoup Paul, n'avait pas pu le lui dire. Sophie a été étonnée de l'impact de cette mort sur sa fille, n'y avait pas prêté attention à l'époque, c'est-à- 
dire quand sa fille avait 9 ans. Elle se sent coupable de ne pas s'être rendue compte de l'importance de Paul pour sa fille.

Pendant ce récit, je m'interroge sur ce Paul. Qui est-il? Je me sens confus et me demande s'il s'agit du compagnon d'une proche cousine de ma patiente mort accidentellement ou bien de l'enfant de 3 ans d'une de ses amies, mort lui aussi dans un accident. À ce moment, je ne sais plus le prénom pourtant différent de l'un et de l'autre. Assez vite cependant et avec clarté, les deux prénoms me reviennent et je réalise que Sophie parle du compagnon de sa cousine, dont on peut penser qu'il a pu représenter une figure déplacée du père odipien pour sa fille. Sophie parlait donc d'un enfant, sa fille, à laquelle elle s'identifie, qui souffre de la mort d'un adulte. Mais, lors de mon accident de pensée, un mouvement inverse et croisé avec son discours manifeste m'a fait vivre une scène où un enfant en latence ou bien un adulte est attristé par la mort d'un petit enfant de 3 ans. Un moment d'ajustement en double (César et Sara Botella, 1984) se révèle dans le transfert-contre-transfert où la figure de l'enfant mort surgit.

Ne serait-ce pas l'enfant de 3 ans en elle, mort psychiquement du fait de l'abandon par ses parents? Mais moi-même ne m'abandonne-t-elle pas, me laissant me perdre dans son histoire? Bien sûr, c'est aussi le père œedipien endossé par le contretransfert qui souffre de la perte de l'enfant, s'opposant à l'indifférence si blessante du père qu'elle m'a rapportée. Ici, je ferais l'hypothèse que les temporalités lors de mon accident de pensée se sont condensées: temps traumatique précoce d'un vécu de mort psychique, temps de ses 9 ans, âge de sa fille lors de la mort de Paul, intemporalité structurelle de l'Edipe.

Je reviens à la séance. Je suis donc troublé et je sors de ce trouble en me disant que ce trouble lui-même est l'indice d'une condensation au-delà du déplacement sur sa fille. Je lui dis alors: "Vous me parlez de Paul, mais de qui s'agit-il vraiment?» Sophie: «Oui...ça me fait penser à mon père, à la déception... je vois mes parents tous les deux, comme des étrangers à moi, dans le chemin, habillés de noir ». Je choisis alors de lui dire: "Comme à leur retour du voyage sans vous, un couple assorti ». Sophie: "Oui, assorti, c'est le mot... Deux tenues semblables, noires et moi en dehors, exclue. Avant, j'étais toujours entre eux, à la place de mon père dans le lit de ma mère ou à celle de ma mère auprès de mon père... là non... j'étais en dehors quand ils étaient partis tous les deux en voyage autour de l'Australie, concevant mon frère et m'envoyant en France ».

\section{Commentaire}

Mon interprétation «un couple assorti» est déterminée par l'option qu'elle a choisie par rapport à ma question, déjà interprétative quant au détour par sa fille, «vous me parlez de Paul mais de qui s'agit-il?» Cette question ouverte pouvait déboucher sur la dimension œedipienne qu'elle a choisie: Paul — mon père, mais aussi sur celle de l'enfant mort : elle petite, témoin prise dans les disputes parentales concernant leur vie sentimentale et sexuelle, elle, abandonnée comme morte, mais aussi elle, identifiée à sa mère enfant (celle-ci avait en effet perdu son père toute 
petite). Le noir figure ce carrefour, noir du deuil du père œedipien, noir du deuil de l'enfant mort, mais aussi noir, couleur de nombre de mes costumes. L'CEdipe a ici fonctionné comme un attracteur face à la problématique traumatique de Sophie. On peut supposer que des indices étaient présents de cette attraction par l'Edipe : l'attente par rapport au père quant au week-end familial, la réception dans le rêve à laquelle elle n'était pas conviée, l'âge de sa fille lors de la mort de Paul, 9 ans, âge auquel elle avait vécu douloureusement la réunion des parents qui l'avait écartée de leur scène intime. Cette fois une scène d'union sexuelle et de procréation. Le désir œdipien pour l'analyste n'est pas interprété, il nécessite d'abord le détour par la reconstruction d'une scène primitive organisatrice, ce qui peut s'effectuer indirectement par la conception de son frère, ce qui concourt à la réparation de la dépression infantile de Sophie.

C'est sans doute cette dynamique qui m’a conduit à privilégier la réunion des parents avec mon interprétation «un couple assorti». Utiliser le mot «assorti » visait en même temps à dépasser l'entente ou non des parents pour mettre l'accent sur leur correspondance ou leur complémentarité au-delà des aléas de l'histoire de leur relation. L'interprétation que j'ai proposée "un couple assorti», au sein d'une construction «comme à leur retour de voyage sans vous» utilisait une formulation verbale secondarisée suffisamment ouverte pour permettre le maximum de possibilités quant au rayonnement associatif chez la patiente. Cette interprétation, par sa relative indétermination, préservait pour l'avenir la possibilité du retour sous d'autres formes des traumatismes précoces et de la dépression infantile qui restaient encore à élaborer dans la cure. Dans l'utilisation qu'en fit Sophie, la structure œedipienne infantile se trouvait privilégiée par rapport aux souvenirs de l'enfance tout en en permettant une certaine symbolisation à travers le deuil nécessaire des objets incestueux. Enfin, cette interprétation laissait ouvert le champ à de possibles associations concernant mon couple; Sophie ayant croisé mon épouse à plusieurs reprises depuis le début de son analyse.

Une autre interprétation eut été possible: « un couple en deuil» qui aurait mis l'accent sur sa dépression infantile, son sentiment d'être morte pour les parents quand ils étaient absents ou absorbés par leurs conflits. Mais outre le fait que cela s'accordait par trop avec son discours manifeste (être «transparente», des parents qui «s'en fichent», etc.), j'y ai renoncé après y avoir songé dans la séance, pensant que stratégiquement l'après-coup d'une scène primitive organisatrice permettrait d'affronter la détresse infantile traumatique et que la douleur du deuil œedipien faciliterait l'abord de son sentiment de déréliction à certaines périodes de ses toutes premières années. Ici, nous voyons comment l'organisateur œedipien chez l'analyste le conduit à intervenir en privilégiant une forme ouverte, potentiellement œdipienne, d'interprétation qui facilitera la fonction attractive de l'Edipe chez la patiente.

Le recours à la problématique œdipienne, induit par Sophie, vient au secours du narcissisme blessé, comme le vêtement de peau qui protège.

Pour terminer, je voudrais souligner que dans cette brève séquence d'analyse, les temporalités et les mouvements transférentiels apparaissent multiples et se conjuguent en montrant les effets de l'après-coup. 
- Un temps traumatique précoce dont témoigne le souvenir-écran de l'abandon par la mère vers 2-3 ans auquel répond la négligence actuelle de son anniversaire par la mère comme la quête d'une peau protectrice. Cet ensemble a valeur d'interprétation symbolique du vécu de détresse précoce. À mon sens, l'actualisation de cette problématique traumatique des tout premiers temps, trace sans souvenir, se fait dans l'accident de pensée contre-transférentiel de l'analyste.

- Un temps odipien infantile que trahissent les souvenirs de la période de latence de ses 9 ans, repris dans l'actualité de la rencontre manquée avec ses parents, qui montre la réinterprétation œedipienne du vécu d'abandon lors des séparations et introduisent à la scène sexuelle des parents dont elle est exclue. La réception à laquelle elle n'est pas conviée est aussi le reflet de la tiercéité du cadre comme de l'abstinence neutre de l'analyste.

- Le temps de l'interprétation-construction qui se situe au temps des souvenirs de la latence et s'appuie sur l'organisation odipienne et le fantasme de scène primitive. La structure est convoquée pour réinterpréter l'histoire, tandis que s'actualise à travers les affects et les mots le vécu d'abandon et d'exclusion et que la perlaboration du deuil des objets œdipiens doit se mettre en œuvre.

\section{Conclusion}

Ces moments de cure que j'ai présentés pourraient conduire à un certain optimisme quant aux ressources psychiques de nos patients et quant à l'avenir du complexe d'Édipe. À partir de mon expérience clinique, tel me semble souvent être le cas. Alors, la cure se déroule dans l'enchaînement et la succession de temps de retour des traumatismes précoces et de leur reprise signifiante dans le réseau symbolique de la conflictualité œedipienne. Cependant les patients qui aujourd'hui viennent trouver un analyste et plus encore qui sont prêts à entreprendre un long travail de cure ne reflètent-ils pas une minorité de sujets en souffrance? Doit-on pour autant penser que seuls les patients ayant une pré-organisation œedipienne suffisamment solide viennent nous trouver et surtout acceptent les contraintes du cadre analytique? Ces situations sont certainement les plus favorables car la rencontre avec les organisateurs œdipiens de l'analyste permet alors l'assomption de la pré-organisation œedipienne de l'analysant. Mais beaucoup de sujets guère organisés selon la structure œedipienne déclinent l'offre de soins ou nous conduisent, quand cela est possible, à adapter notre aide à leurs possibilités psychiques. La reconnaissance du phénomène de transfert et de l'existence d'une altérité chez l'objet corrélative de la croissance du moi sera, dans les meilleurs des cas, une précieuse conquête du travail analytique. Mais nous sommes alors encore bien loin du complexe d'CEdipe.

Christian Seulin

10 , rue Renan

69007 Lyon

France 


\section{Références}

BOTELLA, C., BOTELLA, S., 2001, Le travail du double, in La figurabilité psychique, Delachaux et Niestlé, 1984.

FAIN, M., 1971, Prélude à la vie fantasmatique, Revue française de psychanalyse, 35, 2-3, 291-364.

FREUD, S., 1905/1987, Trois essais sur la théorie sexuelle, traduit de l'allemand par P. Koeppel, Gallimard, Paris.

FREUD, S., 1911/1984, Formulations sur les deux principes du cours des évènements psychiques, in Résultats, idées, problèmes I 1890-1920, traduit de l'allemand, PUF, Paris.

FREUD, S., 1918/1988, À partir de l'histoire d'une névrose infantile, in OC, XIII, traduit de l'allemand, PUF, Paris.

FREUD, S., 1924/1978, La perte de la réalité dans la névrose et dans la psychose, in Névrose, psychose et perversion, traduit de l'allemand, PUF, Paris.

GREEN, A., 1984, Le langage dans la psychanalyse, in Langages, Les belles Lettres.

LAPLANCHE, J., PONTALIS, J.-B., 1985, Fantasme originaire, fantasmes des origines, origines du fantasme, Hachette, 1964.

LE GUEN C., 1974. L'Edipe originaire, Payot, Paris.

ODY, M., 1990, Edipe comme attracteur, in La psychanalyse, questions pour demain, PUF, 1989.

SEULIN, C., 2007, Le complexe d'Edipe dans l'œuvre de Freud, in Actualité de l'CEdipe, PUF, Paris.

WINNICOTT, D.W., 1971/1975, Jeu et réalité, traduit de l'anglais par C. Monod et J-B. Pontalis, Gallimard. 